\title{
Impacto psicológico de niños expuestos a la violencia de forma directa e indirecta de dos casas de protección del ICBF y de una institución educativa de la ciudad de Cali, Colombia'
}

\section{Psychological impact of children exposed to violence directly and indirectly two houses protecting the ICBF and an educational institution in the city of Cali, Colombia}

\author{
María Isabel Posso Chaparro² \\ Mónica Fernanda Chica Velásquez ${ }^{3}$ \\ Universidad Nacional Abierta y a Distancia, UNAD \\ Sandra Mónica Gallego ${ }^{4}$ \\ Universidad ICESI-Santiago de Cali
}

\begin{abstract}
Resumen
Se identifica el impacto psicológico de la exposición indirecta y directa a la violencia, evidenciado en tres cuadros clínicos como son la ansiedad, la depresión y el trastorno de estrés postrauma (TEPT), de 70 menores de ambos sexos, con edades entre los 8 y 12 años pertenecientes a dos casas de protección del $\mathrm{ICBF}^{5}$ y a una institución educativa de la ciudad de Cali. La investigación fue de tipo transversal con un diseño ex post facto retrospectivo. Los instrumentos empleados fueron la Escala revisada de Ansiedad Manifiesta para niños, CMAS-R (Reynold y Richmond, 1978), el Cuestionario Child Depression Inventory, CDI (Kovacks, 1981); el Trauma Symptoms Checklist for Children (TSCC), (Briere, 1996) y el Cuestionario de exposición a violencia (CEV), (Cuevas, Peña y Varela, 2003; Cuevas, 2004). Los resultados de la investigación indican que los niños están siendo víctimas de la exposición a la violencia de manera directa e indirecta, fenómeno que conduce a la pérdida de vidas humanas y a la trasgresión de la integridad física, moral y psicológica de los implicados. Sobre todo de los menores quienes en los últimos tiempos han sido los más afectados, sufriendo como consecuencia un gran impacto psicológico.
\end{abstract}

Palabras clave: violencia, exposición, estrés pos trauma, ansiedad, depresión, niños.

\footnotetext{
Abstract

The psychological impact of indirect and direct exposure to violence, evidenced in three clinical conditions such as anxiety, depression disorder and post-traumatic stress disorder (PTSD), 70 children of both sexes, aged between 8 are identified and 12 years belonging to two houses ICBF protection and an

1 Este estudio fue desarrollado en el marco de la investigación Psicología y Violencia de la Facultad de Humanidades y Ciencias Sociales de la Universidad Javeriana, Cali, coordinado por la Doctora María Clara Cuevas. En el título y desarrollo del texto se reserva el nombre de la institución educativa -en la que se elaboró el trabajo- por solicitud de la entidad.

2 Psicóloga, Especialista en Pedagogía para el Desarrollo del Aprendizaje Autónomo, candidata a Magister de Administración de Organizaciones, énfasis en Gestión Humana. Universidad Nacional Abierta y a Distancia- UNAD; e-mail: maria.posso@unad.edu.co

3 Psicóloga, Especialista en pPedagogía para el Desarrollo del Aprendizaje Autónomo, candidata a Magister de Administración de Organizaciones, énfasis en Gestión Humana. Universidad Nacional Abierta y a Distancia- UNAD; e-mail: monica.chica@unad.edu.co.

4 Psicóloga. Universidad ICESI-Santiago de Cali.

5 El ICBF (Instituto Colombiano de Bienestar Familiar) es una entidad del Estado colombiano, que trabaja por la prevención y protección integral de la primera infancia, la niñez, la adolescencia y el bienestar de las familias en Colombia, para ampliar información se sugiere consultar http://www.icbf.gov.co/portal/page/portal/PortallCBF/Eilnstituto
} 
educational institution in the city of Cali. The research was a cross-sectional retrospective ex post facto design. The instruments used were the revised Manifest Anxiety Scale for Children (Reynold \& Richmond, 1978), the questionnaire Child Depression Inventory, CDI (Kovacks, 1981); the trauma Symptoms Checklist for Children (TSCC), (Briere, 1996) and the Questionnaire and exposure to violence (CEV), (Cuevas, Peña y Varela, 2003; Cuevas, 2004). The research results indicate that children are victims of exposure to violence directly and indirectly, a phenomenon that leads to the loss of human lives and the transgression of physical, moral and psychological integrity of those involved. Especially of minors who in recent times have been hardest hit, suffering as a result a great psychological impact.

Keywords: violence, exposure, post trauma stress, anxiety, depression, children.

Recibido: 31 de julio de 2015 Aceptado: 21 de octubre de 2015

\section{Introducción}

La violencia es un aspecto transversal en la historia de Colombia. La política, la cultura, el entretenimiento y el desarrollo social, han estado enmarcados por manifestaciones de destrucción, vulneración física y emocional de sus habitantes, así como una mutación constante de actores, escenarios y causas.

Los efectos de la violencia se evidencian en cifras como las reportadas por la Unidad de Víctimas en el 2014, donde figuran cerca de 6 millones de personas, la mayoría por desplazamiento forzado y hechos que incluyen amenazas, perdida de algún bien, desaparición, secuestro, actos de terrorismo, homicidios y violencia sexual. Por ejemplo, en el 2012 más de 221.000 personas sufrieron algún hecho de victimización.

De manera específica, la violencia presenta un alto impacto en la población infantil. Las niñas y los niños son testigos de la intimidación, la tortura o la muerte de un ser querido; algunos han visto personas gravemente heridas durante tiroteos indiscriminados o personas víctimas de matanzas; han sido torturados u objeto de amenazas por parte de las fuerzas del conflicto. Otros sufren graves lesiones físicas, amputaciones o quemaduras graves. En determinados lugares, hasta un $10,0 \%$ de los niños presenta una discapacidad física como resultado del conflicto, (Ortiz y Chaskel, 2013, p. 41).

En forma paralela, se presenta otro fenómeno que también está causando estragos en la población infantil y es la exposición a través de los medios de comunicación e internet, que proyectan acciones de violencia, que se consideran indirectas porque los niños no son las víctimas de las lesiones o los daños, si no que están observando cómo estas acciones violentas afectan a otros, como es el caso de los medios y de la observación en el contexto en el que ocurran hechos de violencia.

Sobre esa perspectiva de las incidencias y las realidades a las que se enfrentan los menores, surge el interés de esta investigación, cuyo objetivo principal consistió en aportar elementos para la identificación de las diferencias en el impacto psicológico de niños expuestos a la violencia, de forma directa e indirecta, de dos casas de protección del ICBF y de un colegio de la ciudad de Cali.

La población fue determinada teniendo en cuenta el contexto. Según cifras del Comité Interinstitucional de Muertes Violentas de Santiago de Cali, la ciudad ha registrado altos índices de violencia, como lo evidencia el hecho de que entre el 1 de enero y el 4 de octubre de 2014, se registraron 1.137 muertes violentas. Por otra parte, las condiciones sociales y políticas de la ciudad han contribuido a la creación de grupos al margen de la ley y la vulneración de los derechos humanos, acentuando aún más el fenómeno de la violencia en la comunidad'.

La investigación trabajó con dos grupos; el primero, conformado por 150 niños institucionali-

\footnotetext{
6 Para mayor información sobre las condiciones y los impactos de la violencia en la ciudad, consultar http://www.cali.gov.co/ observatorios/
} 
zados en dos casas de protección del ICBF en la ciudad de Cali, considerados en riesgo, es decir expuestos de manera directa al maltrato físico, psicológico y la negligencia; y el segundo grupo, formado por niños escolarizados de un colegio de la ciudad, con exposición indirecta a la violencia a través de la observación en el contexto o en los medios de comunicación.

\section{Perspectiva teórica}

El marco teórico de la presente investigación aborda el tema de la violencia, su definición y sus manifestaciones, como son la exposición a violencia directa e indirecta.

Asimismo aborda los efectos psicológicos como son: la ansiedad, depresión y trastorno de estrés postraumático, cuadros clínicos que pueden desarrollar los niños y niñas que son expuestos a la violencia tanto de manera directa como indirecta.

\section{Conceptos de violencia}

La violencia es un fenómeno que cada vez cobra más fuerza, al punto de ser hoy uno de los principales medios de resolución de conflictos en la gran mayoría de los países, desencadenando un sin fin de guerras y actos terroristas que lo único que generan son más enfrentamientos violentos, pero sobre todo conducen a la pérdida de vidas humanas y a la trasgresión de la integridad física, moral y psicológica de los implicados. Estos hechos son cada vez más frecuentes y las cifras de víctimas son incalculables, lo que ha despertado el interés institucional y de la academia (UNICEF, 2002; Crouch, J., 2000; Berkowitz 1996; Deas y Gaitán, 1995; entre otros) surgiendo estudios focalizados en explicar e identificar posibles formas de evitar sus alcances en la población que los sufre.

En esa ruta, dichos procesos han dado origen a diversas definiciones de violencia, sin embargo, para fines de la investigación se retomará el concepto de la Organización Mundial de la Salud (OMS), que en en su Informe Mundial
Sobre la Violencia y la Salud, de 2002, define la violencia como...

... "el uso intencional de la fuerza física o el poder, real o por amenaza, contra la persona misma, contra otra persona, o contra un grupo o comunidad que puede resultar en o tiene alta probabilidad de resultar en muerte, lesión, daño psicológico, problemas de desarrollo o de privación."

Según el Plan Migración, Comunicación y Desarrollo (2004), Colombia afronta el conflicto armado más antiguo de América, en el que, aunque toda la población civil sufre por la violencia, son los niños quienes padecen las mayores consecuencias.

\section{Exposición directa a violencia}

La exposición directa a la violencia se refiere a una amplia variedad de situaciones que convierten a los niños en víctimas "directas de la violencia", en tanto que éstos sufren concisamente sus efectos físicos (heridas, muerte, lesiones, etc.) y psicológicos (ansiedad, depresión, trastorno de estrés postraumatico). Este tipo de violencia incluye el maltrato infantil, el desplazamiento forzoso, la participación en la guerra, las heridas con arma de fuego, el secuestro, la muerte de familiares cercanos, el asalto, atraco, robo, entre otros (UNICEF, 2001).

Para fines de la investigación se enfatizó en el maltrato infantil y sus diferentes tipologías, ya que en Colombia los informes estadísticos de organizaciones como el ICBF revelan que en el 2.004, se recibieron más de 2.000 casos de violencia contra niños.

\section{Exposición indirecta a la violencia}

La violencia indirecta podría definirse como la exposición a través de medios indirectos o a situaciones en las que el niño es testigo de acciones violentas, ya sea porque estas acontecen en su propio contexto (lo que se ha denominado violencia comunitaria); no sufren el maltrato físico, psicológico y la negligencia, pero a diario presencian imágenes, situaciones 
o noticias igualmente violentas en los medios de comunicación y en la misma comunidad o contexto en el que viven (Peña y Varela, 2004).

\section{Impacto psicológico de la exposición a violencia directa e indirecta}

Allwood et al. (2002); Garbarino y Kostelny (1996); Goldstein et al. (1997); Pynoos y Nader (1989) plantean que además del nivel de trauma, la duración de exposición a la violencia predice el riesgo para el desarrollo de problemas psiquiátricos en niños.

Así mismo, Crouch (2000) a partir de sus investigaciones describe como los niños que han sido víctimas de maltrato físico, desarrollan una conducta de apego inseguro con sus cuidadores, en comparación con los jóvenes no maltratados. Además, el autor refiere que en los niños que han sido físicamente maltratados también se pueden identificar una serie de dificultades emocionales y comportamentales como ansiedad, depresión, trastornos del sueño, comportamientos autodestructivos, baja autoestima e hiperactividad y dificultades para interpretar el comportamiento de los demás.

En cuanto a los niños que son expuestos a violencia comunitaria, los resultados de la investigación de Mazza y Overstreet (2002) muestran que la exposición a la violencia comunitaria tiene relaciones directas e indirectas con los problemas de salud mental y los comportamientos agresivos y/o antisociales, el trastorno de estrés postraumático, la depresión, el comportamiento suicida y la preocupación.

\section{Materiales y métodos}

La investigación fue de tipo transversal con un diseño ex post facto retrospectivo. La población estuvo conformada por 150 niños y niñas de un colegio y dos hogares del Instituto Colombiano de Bienestar Familiar de la ciudad de Cali, con edades entre los 7 y 12 años, que respondieron afirmativamente a la solicitud de participar en la investigación.
Para fines de la investigación la muestra definitiva estuvo conformada por 70 niños de ambos sexos, con edades entre 8 y 12 años, que se asignaron a la misma de acuerdo a los siguientes criterios de inclusión y exclusión: Se tomaron los sujetos que cumplían los criterios: edad, estrato socioeconómico bajo, consentimiento informado de la institución, de los padres y los mismos niños y que habían sido expuestos a violencia directa e indirecta Se excluyeron aquellos niños con edades inferiores a los 8 años, ya que no cumplían con los criterios para la aplicación del el Trauma Symptoms for Children (TSCC).

Se conformaron dos grupos, cada uno de 35 menores. El primer grupo correspondía a los niños y niñas institucionalizados en dos hogares del ICBF, quienes habían sido evaluados como víctimas de maltrato físico, psicológico o negligencia por un equipo interdisciplinario de dicha institución, el otro grupo lo conformaban los niños de un colegio de la ciudad de Cali, para los que ya habían sido determinados los niveles de exposición a violencia a partir de un estudio previo (Cuevas, 2005).

Los instrumentos empleados fueron la Escala Revisada de Ansiedad Manifiesta para niños, CMAS-R (Reynold y Richmond, 1978), el Cuestionario Child Depression Inventory, CDI (Kovacks, 1981); el Trauma Symptoms Checklist for Children (TSCC) (Briere, 1996) y el Cuestionario de Exposición a Violencia (CEV) (Cuevas, Peña y Varela, 2003; Cuevas, 2004). El cuestionario está compuesto por 13 áreas temáticas correspondientes a fenómenos de la violencia social y política en Colombia: guerrilla, paramilitares, secuestro, personas desplazas por la violencia, atentados terroristas, amenazas, atracos, asaltos con armas, personas heridas con cuchillos o balas, personas asesinadas, masacres, robos e insultos. EI CEV arroja un puntaje general que permite ubicar a cada sujeto en niveles de exposición, alto, medio o bajo.

Luego de la aplicación individual de los instrumentos, se realizaron los análisis estadísticos de los resultados arrojados por las escalas de cada uno de los instrumentos, 
utilizando el paquete estadístico (SPSS) versión 13 para Windows, a partir de dicho análisis se comprobaron las hipótesis planteadas para esta investigación.

\section{Presentación de resultados}

Para la presentación y análisis de resultados, se obtuvieron distribuciones de frecuencia simples para las cuatro pruebas aplicadas, las cuales permiten ser puntuadas y estudiadas como variables en escala de intervalos, razón por la que fue necesario utilizar métodos propios de la estadística no paramétrica para el análisis de los datos.

Las variables generadas en las diferentes respuestas de los cuestionarios TSCC, CMAS-R y $C D I$ se cruzaron con las variables edad e institución en la que se llevó a cabo la aplicación. Se usó un alfa de 0.05 para establecer significancia estadística.

Para la organización de los resultados, se presentan unos datos generales, que se refieren a las características sociodemográficas de la muestra edad y sexo de los dos grupos que conformaron la muestra. Esta diferenciación entre los dos grupos de edad se realizó teniendo en cuenta la frecuencia que se denota en el grupo de niños con edades comprendidas entre los 11 y 12 años.

El primer grupo, niños institucionalizados en dos hogares de protección del ICBF, se encuentra conformado por 35 niños todos pertenecientes al género masculino, con edades entre los 8 y 12 años.

El segundo, lo componen 35 niños (17 niñas y 18 niños), pertenecientes a un colegio de la ciudad con edades entre los 8 y 12 años.

Posteriormente, se presentan los datos especíicos en los que se realiza inicialmente una diferenciación entre los niños que son expuestos a violencia directa e indirecta. Quedando establecido que los niños del ICBF son expuestos a violencia de manera directa y los niños del colegio son expuestos a violencia indirecta.

\section{Exposición a violencia}

Tabla 1. Distribución de instituciones por niveles de exposición a violencia y victimización.

\begin{tabular}{|c|c|c|c|}
\hline & & ICBF & COLEGIO \\
\hline & NIVEL & $\%$ & $\%$ \\
\hline \multirow{5}{*}{ EXPOSICION } & BAJO & 42,8 & 84,3 \\
\hline & MEDIO & 5,7 & 10,0 \\
\hline & MODERADA & 0 & 2,9 \\
\hline & SEVERA & 1,4 & 1,4 \\
\hline & TOTAL & 100,0 & 100,0 \\
\hline \multirow{5}{*}{ VICTIMIZACION } & BAJO & 7,1 & 20,0 \\
\hline & MEDIO & 1,4 & 0 \\
\hline & SEVERO & 0 & 0 \\
\hline & $\begin{array}{l}\text { NO HA SIDO } \\
\text { VICTIMA }\end{array}$ & 41,4 & 78,6 \\
\hline & TOTAL & 100,0 & 100,0 \\
\hline
\end{tabular}

La Tabla 1 permite observar el nivel de exposición a la violencia y el nivel de victimización de los dos grupos participantes. Como puede observarse, el 1,4\% tanto de participantes pertenecientes al ICBF como el correspondiente al colegio, presentan exposición a violencia a nivel severo. En cuanto a la victimización, se puede notar que no se presentó a nivel severo en ninguno de los dos grupos. Sin embargo, los participantes del ICBF presentan mayor porcentaje a nivel bajo y medio que los pertenecientes al colegio.

\section{Trastorno de estrés post trauma}

A continuación se presentan los resultados obtenidos a partir del cuestionario TSCC el cual indaga la presencia de trastorno de estrés postrauma. 
Tabla 2. Distribución de frecuencia TSCC por cuadros escalas e institución.

\begin{tabular}{|c|c|c|c|}
\hline ESCALAS & NIVEL & $\begin{array}{c}\text { ICBF } \\
\%\end{array}$ & $\begin{array}{c}\text { COLEGIO } \\
\%\end{array}$ \\
\hline \multirow{4}{*}{ Ansiedad } & Normal & 17,1 & 71,4 \\
\hline & Leve & 62,9 & 20,0 \\
\hline & severo & 20,0 & 8,6 \\
\hline & TOTAL & 100,0 & 100,0 \\
\hline \multirow{4}{*}{ Depresión } & Normal & 25,7 & 54,3 \\
\hline & Leve & 68,6 & 28,6 \\
\hline & Severo & 5,7 & 17,1 \\
\hline & TOTAL & 100,0 & 100,0 \\
\hline \multirow{4}{*}{ Ira } & Normal & 62,9 & 85,7 \\
\hline & Leve & 37,1 & 11,4 \\
\hline & Severo & 0 & 2,9 \\
\hline & TOTAL & 100,0 & 100,0 \\
\hline \multirow{4}{*}{ Disociación } & Normal & 28,60 & 97,1 \\
\hline & Leve & 62,9 & 2,9 \\
\hline & Severo & 8,6 & 0 \\
\hline & TOTAL & 100,0 & 100,0 \\
\hline \multirow{4}{*}{ Disociación-o } & Normal & 20,0 & 77,1 \\
\hline & Leve & 65,7 & 22,9 \\
\hline & Severo & 14,3 & 0 \\
\hline & TOTAL & 100,0 & 100,0 \\
\hline \multirow{4}{*}{ Disociación-f } & Normal & 20,00 & 62,9 \\
\hline & Leve & 34,3 & 34,3 \\
\hline & Severo & 45,7 & 2,9 \\
\hline & TOTAL & 100,0 & 100,0 \\
\hline \multirow{4}{*}{ TEPT } & Normal & 40,00 & 71,4 \\
\hline & Leve & 54,3 & 20,0 \\
\hline & Severo & 5,7 & 8,6 \\
\hline & TOTAL & 100,0 & 100,0 \\
\hline
\end{tabular}

Como se aprecia en la Tabla 2, el $20 \%$ del grupo perteneciente a las instituciones del ICBF en esta prueba, se ubica en ansiedad severa, en comparación con el colegio, donde un $8.6 \%$ de la muestra presenta ansiedad a este mismo nivel.

Del grupo del ICBF, un $62.9 \%$ presenta un nivel leve de ansiedad, en relación con el otro grupo en el cual el $20 \%$ presenta ansiedad a un nivel leve.

Con respecto a la depresión, se encontraron diferencias ya que en el grupo del ICBF, el $5.7 \%$ presenta un nivel severo, en comparación con el grupo del colegio en el que el $17.1 \%$ presenta depresión a este mismo nivel.
En cuanto a la ira, se encontró que ningún sujeto del grupo del ICBF se encuentra en un nivel severo, en comparación con el grupo del colegio, en el cual el $2.9 \%$ se ubica en un nivel severo para dicho cuadro clínico.

Así mismo, teniendo en cuenta el número total de niños del ICBF, se encontró que un porcentaje representativo, correspondiente al $62.9 \%$, presenta un nivel leve de disociación, en relación con el grupo del colegio, en donde solo el $2.9 \%$ presentó este cuadro clínico a un nivel leve.

Finalmente, en lo que respecta TEPT, se observó que el $5.7 \%$ del grupo conformado por los niños del ICBF, presenta un nivel severo para esta sintomatología, en comparación con el grupo conformado por niños del colegio, en el cual el $8.6 \%$ se ubica en este nivel. De igual manera, se observó que el $54.3 \%$ de los niños del ICBF presenta un nivel leve de TEPT, al contrario de los niños del colegio en donde solo el $20 \%$ se ubica en este nivel.

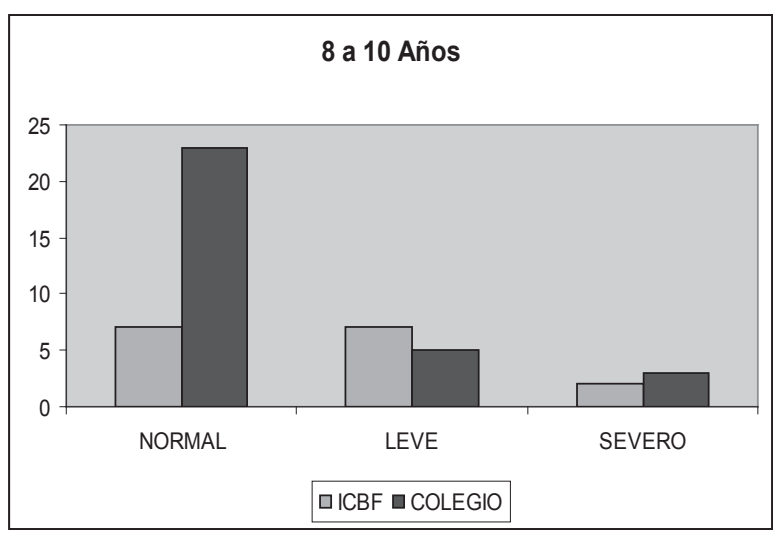

Figura 1. Presencia de TEPT. Por edad e institución (8 a 10 años).

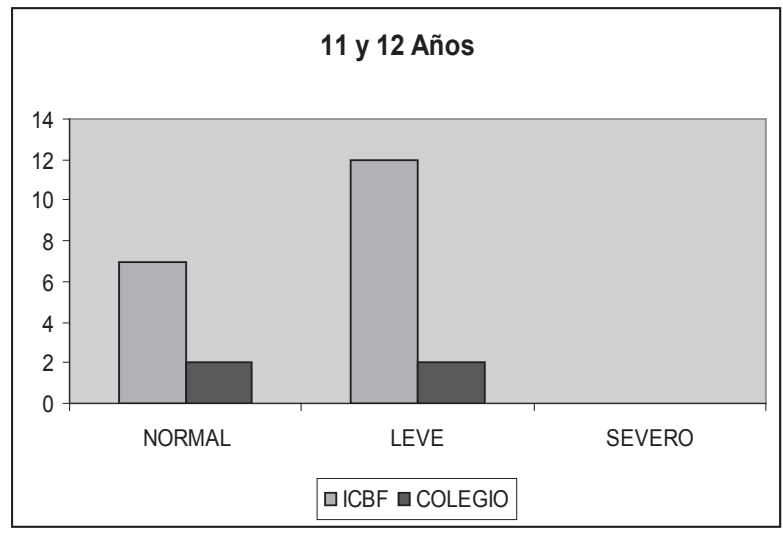

Figura 2. Presencia de TEPT. Por edad e institución (11 y 12 años). 
Las figuras 1 y 2 indican la presencia de estrés post traumático según la edad de los sujetos de ambos grupos. En cuanto a los sujetos con edades entre 8 y 10 años, el $10.0 \%$ de los sujetos del ICBF presentan el trastorno en un nivel leve, respecto a los sujetos del colegio en el mismo rango de edad, de los cuales el $7.1 \%$ presentan el cuadro clínico a dicho nivel. El $2.9 \%$ de los sujetos del ICBF presentan un nivel severo de TEPT a diferencia del colegio en donde el $4.3 \%$ de los sujetos presenta el trastorno a este nivel.

En relación a los sujetos de 11 y 12 años, el 17,1 $\%$ de los sujetos del ICBF presentan un nivel leve del trastorno en relación con el colegio en donde solo el 2,9\% de los sujetos presentaron el cuadro clínico a dicho nivel. Finalmente, ninguno de los sujetos de ambos grupos presentó un nivel severo del cuadro clínico.

\section{Ansiedad}

Después de revisar los resultados del TSCC se procede a revisar la presencia de ansiedad teniendo en cuenta los datos arrojados por el CMAS-R.

Tabla 3. Distribución de frecuencia CMAS-R por categorías e institución.

\begin{tabular}{|l|l|r|r|}
\hline \multirow{2}{*}{ CATEGORIAS } & NIVEL & ICBF \% & $\begin{array}{c}\text { COLEGIO } \\
\text { \% }\end{array}$ \\
\hline \multirow{4}{*}{$\begin{array}{l}\text { Ansiedad } \\
\text { fisiológica }\end{array}$} & Normal & 60,0 & $\mathbf{8 2 , 9}$ \\
\cline { 2 - 4 } & Leve & 25,7 & $\mathbf{1 7 , 1}$ \\
\cline { 2 - 4 } & severo & 14,3 & $\mathbf{0}$ \\
\cline { 2 - 4 } & TOTAL & $\mathbf{1 0 0 , 0}$ & $\mathbf{1 0 0 , 0}$ \\
\hline \multirow{4}{*}{ Inquietud } & Normal & 65,7 & $\mathbf{8 5 , 7}$ \\
\cline { 2 - 4 } & Leve & 22,9 & $\mathbf{1 1 , 4}$ \\
\cline { 2 - 4 } & Severo & 11,4 & $\mathbf{2 , 9}$ \\
\cline { 2 - 4 } & TOTAL & $\mathbf{1 0 0 , 0}$ & $\mathbf{1 0 0 , 0}$ \\
\hline \multirow{4}{*}{ Preocupación } & Normal & 54,3 & $\mathbf{8 0 , 0}$ \\
\cline { 2 - 4 } & Leve & 42,9 & $\mathbf{1 7 , 1}$ \\
\cline { 2 - 4 } & Severo & 2,9 & $\mathbf{2 , 9}$ \\
\cline { 2 - 4 } & TOTAL & $\mathbf{1 0 0 , 0}$ & $\mathbf{1 0 0 , 0}$ \\
\hline \multirow{4}{*}{$\begin{array}{l}\text { ANSIEDAD } \\
\text { TOTAL }\end{array}$} & Normal & 37,1 & $\mathbf{8 0 , 0}$ \\
\cline { 2 - 4 } & Leve & 51,4 & $\mathbf{1 1 , 4}$ \\
\cline { 2 - 4 } & Severo & 8,6 & $\mathbf{2 , 9}$ \\
\cline { 2 - 4 } & No Presenta & 2,9 & $\mathbf{5 , 7}$ \\
\cline { 2 - 4 } & TOTAL & $\mathbf{1 0 0 , 0}$ & $\mathbf{1 0 0 , 0}$ \\
\hline
\end{tabular}

En la tabla 3 se observa que el 14,3\% del grupo perteneciente a las instituciones del ICBF, presenta un nivel de ansiedad fisiológica severa en comparación con el colegio, donde no se presenta, En la categoría inquietud, el grupo del ICBF presenta un nivel severo con un $11,4 \%$ en comparación con el colegio que presenta un 2,9 $\%$ y en relación a la categoría preocupación el grupo de las instituciones del ICBF presentan un nivel severo igual que el grupo del colegio.

La categoría ansiedad total del grupo de las instituciones del ICBF presenta un $8,6 \%$ de nivel severo en comparación a la ansiedad total del grupo del colegio que presenta un nivel severo de $5,7 \%$.

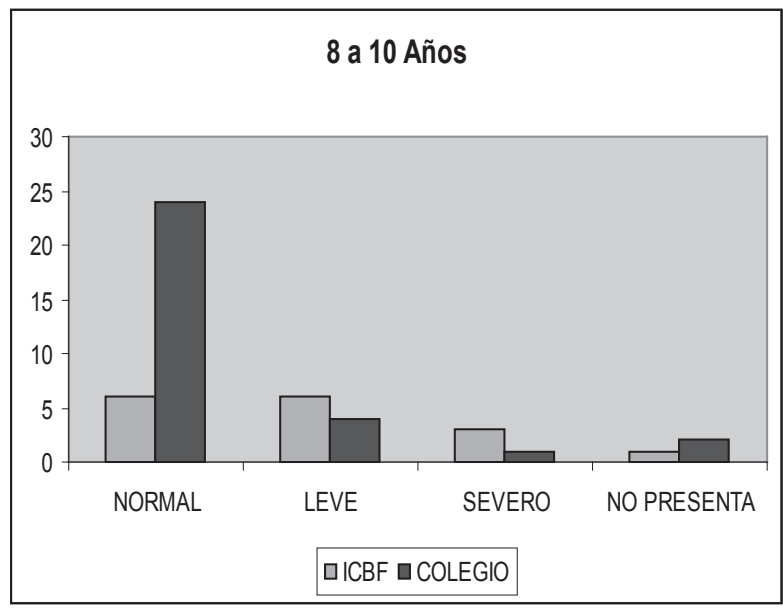

Figura 3. Presencia de ansiedad. Por edad e institución según CMAS-R (8 a 10 años).

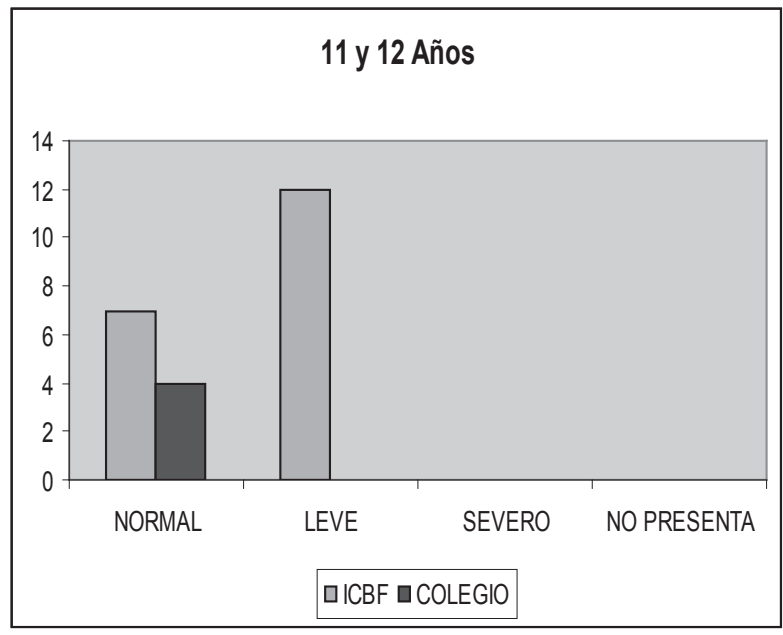

Figura 4. Presencia de ansiedad por edad e institución según CMAS-R (11 y 12 años). 
Las figuras 3 y 4 muestran la presencia de ansiedad a partir de la edad y la institución, es decir, ICBF o colegio. En el rango de edad de los 8 a 10 años, se observa que el $8,7 \%$ de los sujetos del ICBF y el $5,7 \%$ de los sujetos del colegio presentan un nivel leve de ansiedad. En cuanto al nivel severo $4,3 \%$ de los sujetos del ICBF y el 1,4 $\%$ del colegio obtuvieron puntuaciones elevadas en dicho cuadro clínico.

Respecto a la edad comprendida entre los 11 y 12 años $17,1 \%$ de los sujetos del ICBF presentaron un nivel de ansiedad leve en comparación con el colegio en donde ningún sujeto presentó ansiedad a dicho nivel. A diferencia del grupo de sujetos con edades entre 8 y 10 años, ninguno de los sujetos de 11 y 12 años obtuvo niveles severos de ansiedad.

\section{Depresión}

A continuación se realiza la presentación de los datos arrojados por el cuestionario CDI el cual evalúa la presencia del cuadro clínico depresión.

Tabla 4. Distribución de frecuencia CDI por categorías e institución.

\begin{tabular}{|l|l|r|r|}
\hline & NIVEL & \multicolumn{1}{c|}{$\begin{array}{c}\text { ICBF } \\
\%\end{array}$} & \multicolumn{2}{c|}{$\begin{array}{c}\text { COLEGIO } \\
\text { \% }\end{array}$} \\
\hline \multirow{3}{*}{ DEPRESION } & Normal & 54,3 & $\mathbf{8 5 , 7}$ \\
\cline { 2 - 4 } & Leve & 28,6 & $\mathbf{5 , 7}$ \\
\cline { 2 - 4 } & Severo & 17,1 & $\mathbf{8 , 6}$ \\
\cline { 2 - 4 } & TOTAL & $\mathbf{1 0 0 , 0}$ & $\mathbf{1 0 0 , 0}$ \\
\hline
\end{tabular}

Como se puede observar en la tabla 4 el 17.1 $\%$ del grupo conformado por los niños del ICBF se ubica en un nivel severo para depresión, en comparación con el grupo conformado por los niños del colegio, en el cual el $8.6 \%$ de estos se encuentra en un nivel severo de depresión.

En las figuras 5 y 6 se aprecia la presencia de depresión a partir de la edad y la institución, es decir ICBF o colegio. En el rango de edad de los 8 a 10 años, se observa que el 5,7\% de los sujetos del ICBF y el 2,9\% del colegio presentan

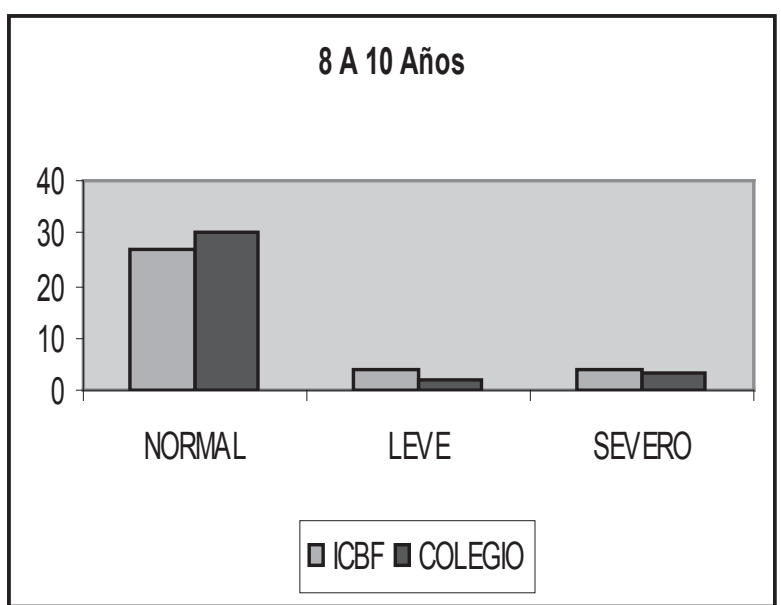

Figura 5. Presencia de depresión (8 a 10 años).

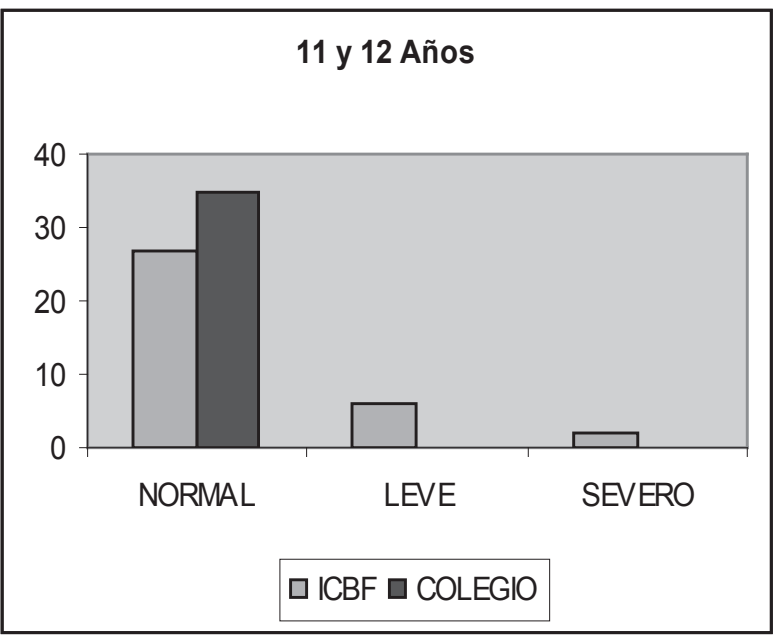

Figura 6. Presencia de depresión por edad e institución. Por edad e institución (11 y 12 años).

un nivel leve de sintomatología depresiva. En cuanto al nivel severo el 5,7\% de los sujetos del ICBF y el 4,3\% de los sujetos del colegio obtuvieron puntuaciones significativas en dicho cuadro clínico.

Referente a la edad comprendida entre los 11 y 12 años el 8,6\% de los sujetos del ICBF presentaron un nivel de depresión leve a diferencia del colegio en donde ningún sujeto presento depresión a dicho nivel. Asimismo el 2,9\% de los sujetos del ICBF presento depresión a un nivel severo, en contraste del colegio en donde no se presentó niveles severos. 


\section{Discusión}

La presente investigación tuvo como objetivo identificar el impacto psicológico de la exposición indirecta y directa a la violencia, evidenciado en tres cuadros clínicos como son la ansiedad, la depresión y el trastorno de estrés postrauma (TEPT).

Encuanto al impacto psicológico de la exposición indirecta a la violencia en los niños de ambos grupos (grupo 1: ICBF y grupo 2: colegio), se determinó su nivel de exposición y victimización a partir de cuatro fenómenos: guerrilla, secuestro, amenazas, personas heridas con arma blanca o bala, de acuerdo al Cuestionario de exposición a violencia (CEV). Cabe resaltar que estos cuatro fenómenos se seleccionaron debido al alto nivel de prevalencia en ambos grupos, caracterizando el conocimiento de estos fenómenos, las fuentes de información y las experiencias de personas víctimas de hechos violentos. Respecto al nivel general de la exposición indirecta a la violencia, los resultados muestran que no existen diferencias significativas entre los dos grupos, puesto que existen puntuaciones similares entre ambos.

Se puede decir entonces que los niños tienen un conocimiento de los fenómenos que hacen parte de la violencia indirecta. El mayor conocimiento corresponde a guerrilla, seguido en su orden por el secuestro, amenazas y personas heridas con arma blanca o bala. Este hallazgo puede ser consecuencia de la alta exposición de los niños a la violencia a través de los medios masivos de comunicación. Al respecto los resultados indican que la televisión y el periódico constituyen las principales fuentes de información a través de los cuáles los niños han obtenido conocimiento en cuanto a los fenómenos de la violencia mencionados. Varios estudios se han centrado en la influencia de estos medios de comunicación en el comportamiento violento de los niños, además de otras repercusiones psicológicas, (Berkowitz 1996; García 2000; Mayor 2001). De igual manera, el estudio realizado por Gil (2005), destaca que los niños y adolescentes son más agresivos en cuanto más televisión ven, independiente de si los contenidos son o no violentos.
Sin embargo, es importante resaltar que, según los resultados, los niños del grupo 2 obtuvieron mayor puntaje en la opción televisión y periódico, en cuanto a las fuentes de información de los cuatro fenómenos.

No obstante, la familia constituye una importante fuente de información sobre estos fenómenos violentos, lo que podría deberse a la situación por la que actualmente atraviesa el país, lo que obliga o influye a que estos temas sean tratados con mayor frecuencia en las conversaciones familiares. Cabe resaltar que la información proveniente de la familia es mayor en el grupo 2, esto podría deberse a que estos niños no han tenido rupturas familiares y conviven actualmente con sus familias, a diferencia del grupo 1, los cuales se encuentran institucionalizados, quienes, a diferencia del grupo 2, presentaron puntuaciones altas en la opción amigos para fuentes de información, debido a que este es el núcleo con el que interaccionan con mayor frecuencia y con el que además comparten experiencias.

Porotra parte, existe un alto nivel de conocimiento de experiencias de personas que han sido víctimas de estos fenómenos violentos. Con mayor frecuencia los niños conocen un familiar que ha vivido alguna experiencia relacionada con guerrilla, secuestro, amenazas y personas heridas con arma blanca o bala; sin embargo, el grupo 1 obtuvo puntajes más elevados en esta opción, esto podría deberse a que esta población ha sido más vulnerable a las manifestaciones de violencia en su contexto inmediato desde sus primeros años de vida, en este sentido, estos niños pueden ser más sensibles a desarrollar una amplia gama de trastornos psicológicos, tal como lo menciona Wallach (1996), al plantear que los primeros años de vida son los más críticos en la preparación para el desarrollo futuro del niño, las experiencias durante estos años son muy importantes para el crecimiento saludable de los niños, por tanto la violencia ejerce un efecto negativo.

Así mismo, los niños pueden presentar problemas en la conducta y síntomas importantes de estrés postraumático y depresión, esto se evidencia en 
los resultados obtenidos, debido a que algunos de los niños que presentaron puntuaciones elevadas en estos trastornos también obtuvieron niveles altos en cuanto a la exposición a la violencia. En esta medida queda demostrado el estudio realizado por Vermeiren et. AI (2002), en el que indicó que los niños que se enfrentan a la exposición de altos niveles de violencia están en riesgo de presentar sintomatología depresiva y comportamiento agresivo.

En cuanto a los cuadros clínicos ansiedad, depresión y trastorno de estrés pos trauma, en los resultados arrojados en el presente estudio se identificó que los síntomas más frecuentes de ansiedad reportados por los niños son aquellos tales como la preocupación permanente por las cosas, la inquietud y el miedo a que algo malo pueda ocurrir, los que dan cuenta de este cuadro clínico, lo que se relaciona con la definición dada por Rojas (1998), en la cual indica que la ansiedad se caracteriza por inquietud interior, desasosiego, inseguridad, presentimiento de la nada, temores difusos, anticipación de lo peor, estado de alerta, tensión motora, expectación negativa, temor a la muerte, temor a la locura, temor a perder el control, temor a suicidarse y quejas somáticas como: dolor precordial, sequedad en la boca, dificultad respiratoria, escalofríos, manos sudorosas, sensación de mareo o vértigo y micciones frecuentes.

De la misma manera, diversos autores como Campo, Cogollo, Díaz (2002), Martínez, Richters y Valla (1990), citados por Berman, Kurtines, Silverman (2000), plantearon a través de sus investigaciones la aparición de ansiedad e indefensión en relación con haber sido víctimas de violencia (maltrato infantil, el desplazamiento forzoso, la participación en la guerra, las heridas con arma de fuego, el secuestro, la muerte de familiares cercanos, el asalto, atraco y robo, entre otros) o de algún acto violento ocurrido de manera indirecta.

En cuanto al cuadro clínico ansiedad, en la presente investigación se obtuvieron indicadores clínicos significativos, en especial para el grupo conformado por los niños del grupo 1, de los cuales 3 niños se ubican en un nivel severo y 18 en un nivel leve. Esto puede relacionarse con los resultados obtenidos de la investigación realizada por Crouch (2000), en la cual esclarece que en los niños que han sido víctimas de maltrato físico pueden encontrarse indicadores de dificultades emocionales como ansiedad depresión y trastornos del sueño. También en el estudio realizado por Cogollo, Díaz, Campo (2002), en 41 niños y niñas entre los 6 y 12 años de edad provenientes de hogares desplazados de Cartagena, se encontró que 39 de ellos necesitan una exploración cuidadosa de los síntomas. Por otra parte Allwod et. al (2002), Gabardino y Kostelny (1996), Goldstein et. Al (1997), Macksoud (1992), Nader y Pynnos (1989), plantean que, además del nivel de trauma, la duración de la exposición a la violencia predice el riego para el desarrollo de problemas psiquiátricos en niños.

Partiendo de lo anterior podría explicarse de esta manera la diferencia de la ubicación en los niveles entre los dos grupos. Sin embargo, en los niños pertenecientes al grupo 2 se encontró que, en menor proporción, algunos se ubicaron en el nivel severo, esto podría deberse a que estos niños han estado expuestos de manera indirecta a la violencia, así lo demuestran los estudios realizados por el Center on Media and Child Health del Hospital Infantil de Boston (2003), publicado en el 2005 por Kaise Family Foundation en la Zenit, en la cual llegan a la conclusión de que los medios de comunicación contribuyen a la ansiedad, falta de sensibilidad y aumento de la agresión.

En cuanto a la presencia del cuadro clínico de depresión, según Rojas (1998), esta es definida como un descenso del estado de ánimo, inhibición, excitación, poco apetito o pérdida de peso, trastornos del ritmo del sueño, trastornos en la esfera de la memoria, fatiga, cansancio enorme, quejas somáticas frecuentes como: dolores diversos, molestias digestivas, síntomas cardiovasculares y respiratorios, perdida del interés por las actividades habituales.

En este estudio se encontró que el grupo 2 se ubica en mayor proporción en el nivel normal $y$, que los niños del grupo 1 lo hacen más en los 
niveles leve y severo, en comparación con los niños del grupo 2.

A partir de esto podría explicarse el porqué de la ubicación de los niños del grupo 1 en niveles severo y leve, si tenemos en cuenta que son niños que han estado expuestos con mayor frecuencia a la violencia de forma directa, esto podría corroborarse en la investigación realizado por Rodríguez (2003), con 42 niños de 8 a 18 años, los resultado de dicha investigación indicaron que evidentemente los niños que habían sido víctimas de maltrato y de castigo físico severo presentaban sintomatología depresiva.

Finalmente, en cuanto al TEPT, Ardila y Santacruz (2002) plantean que

\begin{abstract}
...este trastorno surge como una respuesta diferida a una situación breve o duradera de naturaleza catastrófica que causaría, por si misma, sufrimiento y consternación en cualquier persona, refieren que los signos y síntomas del TEPT son: episodios reiterados de reviviscencia del trauma en vigilia y en sueños, evitación de actividades y situaciones que evoquen el trauma, sensación de embotamiento emocional, de desapego de los demás y anhedonia, pueden presentarse episodios agudos con características de pánico o agresividad, a consecuencia de un estímulo evocativo del trauma o de la reacción frente a él, hay un estado de hipervigilancia, un incremento de la respuesta de sobresalto e insomnio, a veces pertinaz. (p. 462).
\end{abstract}

Cabe mencionar el hallazgo reportado por Ayala y De Paúl (2004) en el que afirman que las personas que han sido víctimas de situaciones traumáticas son más vulnerables a presentar TEPT. Esto se hace evidente en los resultados del estudio, en el que se encontraron diferencias entre ambos grupos de la muestra, puesto que el grupo 1, conformado por 35 niños y niñas, de los cuales el $60 \%$ cumple con los criterios diagnósticos del TEPT, ya sea a un nivel leve o severo, a diferencia del grupo 2, de igual número de participantes del grupo anterior, del que solo el $28,6 \%$ presenta el cuadro clínico. Así se evidencia como los niños que son víctimas directas de la violencia de diferentes formas muestran una prevalencia de síntomas de TEPT, no obstante, cabe mencionar, tal como lo demuestra el estudio de los autores, la severidad del cuadro clínico depende del tipo de trauma sufrido, género, edad y la presencia de otros cuadros clínicos. Lo anterior se refleja en los resultados obtenidos, ya que los niños con edades entre los 8 y 10 años de ambos grupos de la muestra, superaban al grupo con edades equivalentes a los 11 y 12 años en la presencia del cuadro clínico de TEPT.

Se destaca cómo el fenómeno de la violencia tiene una incidencia significativa en la edad para el desencadenamiento del TEPT como cuadro clínico, sin dejar de lado los demás factores mencionados, que también pueden influir en su desarrollo. De esta forma se destacan los resultados obtenidos en donde 16 sujetos del grupo 1 que presentaron TEPT también obtuvieron puntuaciones significativas en los cuadros clínicos de depresión y ansiedad, hallazgo que no puede pasar desapercibido teniendo en cuenta las posibles repercusiones de la no detección e intervención temprana. Es de relevancia mencionar que al establecer una relación entre ambos grupos, la comorbilidad con otros cuadros clínicos no fue tan frecuente en el grupo 2, en donde solo 5 sujetos de este grupo cumple con los criterios diagnósticos de ansiedad, depresión y TEPT, o están propensos a desarrollarlos. Aunque esta cifra no es tan significativa en comparación con la del grupo 1 , también es de total preocupación ya que da cuenta de qué tan severo es el impacto de la exposición a violencia en niños, ya sea de forma directa o indirecta.

Como se puede apreciar a través de la discusión, los resultados más significativos encontrados en la investigación son el impacto psicológico que tiene la exposición a la violencia de manera directa e indirecta. Aunque se encontraron diferencias entre los dos grupos, resaltándose generalmente las puntuación del grupo 1, es relevante mencionar que en el grupo 2 también se evidenciaron puntuaciones significativas, lo que da cuenta que la exposición a la violencia de manera indirecta también está causando impacto en los niños. 


\section{Conclusiones}

Los resultados de la investigación indicaron que los niños están siendo víctimas de la exposición a la violencia de manera directa e indirecta, fenómeno que conduce a la pérdida de vidas humanas y a la trasgresión de la integridad física, moral y psicológica de los implicados, sobre todo de los menores, quienes en los últimos tiempos han sido los más afectados, sufriendo como consecuencia un gran impacto psicológico.

En cuanto a lo anterior, se halló que el grupo conformado por los niños del ICBF (grupo 1), se encuentra expuesto de manera directa a la violencia, ya que obtuvieron puntuaciones más altas en la categoría victimización, a diferencia del grupo conformado por los niños del colegio (grupo 2), quienes obtuvieron una puntuación más alta en la categoría exposición, esto podría deberse al fácil acceso de los niños del grupo 2 a la televisión y a otros medios que muestran información acerca de la violencia. Los datos anteriores fueron los resultados encontrados a través del cuestionario de exposición a la violencia (CEV).

Igualmente, en referencia a los cuadros clínicos ansiedad, depresión y TEPT considerados como posible impacto de la exposición a violencia directa e indirecta, se presentaron en altas proporciones, destacándose el grupo 1 en comparación al grupo 2. Esto podría deberse a que el grupo 1 ha estado expuesto de manera directa a la violencia, y ha tenido que vivenciar hechos violentos con mayor frecuencia que el grupo 2.Sin embargo, en el grupo 2 se pudieron observar también algunas puntuaciones significativas, debido a que estos niños también han estado expuestos a violencia de manera indirecta y han tenido conocimiento de ésta, aunque de manera diferente, o han vivenciado la violencia, aunque en menor magnitud.

Por otra parte, aunque no se identificó que los niños que habían sido expuestos a violencia indirecta presentaran puntuaciones altas en la sintomatología de los cuadros clínicos, si existe un impacto en los niños como consecuencia de esta exposición manifestada en reacciones como miedo, tristeza y preocupación, que reportaron los niños de este grupo. Estas reacciones cons- tituyen un efecto en el bienestar del niño, que los hace vulnerables al desarrollo de trastornos psicológicos.

Igualmente, un alto porcentaje de niños han sido testigos de fenómenos violentos, lo que es considerado como un factor de riesgo para la aparición de los cuadros clínicos revisados en el presente estudio, debido a la intensidad del fenómeno y la cercanía al mismo.

Esta investigación es un aporte significativo para la psicología ya que se evidenció que si existe un impacto en los niños como consecuencia de la exposición a la violencia ya sea de manera directa o indirecta. Por tal motivo, es pertinente el profundizar sobre el tema con el fin de proponer intervenciones oportunas que logre reducir tal impacto, que no solo se manifiesta a corto plazo si no a largo plazo, ya que como lo indican los resultados, es evidente que todos los niños son vulnerables a desarrollar cuadros clínicos de este tipo.

\section{Referencias}

Abello R., Amar J., Botto A., Carrillo R., Castro Y., Linares E., y Racedo H. (2001). Efectos de la violencia política sobre las emociones de ira, miedo y ansiedad en niños colombianos de 11 y 12 años. Recuperado el 14 de julio de 2005 de http://www. rlpsi.org/avances/volumen 19

Angell RH, Kinzie, JD., Manson S., Rath B. y Sack, WH. (1996). The psychiatrict effects of massive trauma on Cambodian children. En W. Fremont. Childhood reactions to terrorism-induced trauma: a review of the past 10 years. Journal of the American academy of child \& adolescent psychiatric, 43, 381392. Recuperado el 22 de Julio de http:// www. jaacap.com/ -

Albisu M., Carrascosa A., Clemente M., García-Reyna N., Gussinyer S., Gussinyer M., Yeste D., Vicens E. (sf). Programa para el tratamiento integral de la obesidad denominadoniñ@s en movimiento. Recuperado el 10 de enero de 2006 dewww.seep. es/privado/download.asp?url=congresos/C20.

Allwood, M. A., Bell-Dolan, D., y Arshad, S. (2002). Children's trauma and adjustment reactions to violent and nonviolent war experiences. Journal of the American academy of child \& adolescent psychiatric, 41. Recuperado el 22 de julio de http:// www.jaacap.com/ - 
American Academy of Child e Adolescent Psychiatric. (1999). Los niños y la violencia en televisión. Recuperado el 14 de julio de 2005 de http://www. aacap.org/publications/opntsfam/violence

American Psychological Association (2002). Manual de estilo de publicaciones de la American Psychological Association (2a. Ed.) México, D.F.: El Manual Moderno

Aran S., Barata F., Busquet J., Medina P., y Moron S. (2003) Infancia, violencia y televisión: usos televisivos y percepción infantil de la violencia en la televisión. Barcelona:Trípodos.

Ardila, S., y Santacruz, H. (2002). Trastorno de estrés postraumático. En C. Gómez-Restrepo, G. Hernández, A. Rojas, H. Santacruz. Fundamentos de psiquiatría clínica: niños, adolescentes y adultos. Colombia: CEJA, Centro Editorial Javeriano. 460-467

Arruabarrena, M., y De Paúl, J. (1997). Maltrato a los niños en la familia: evaluación y tratamiento. Madrid: ediciones Pirámide.

Arredondo, R., y Damián, D. Martínez, J., y Reyes, C. (2005). Efectos psicológicos ocasionados por los programas televisivos a niños entre 5 y 13 años de edad. Recuperado el 21 de julio de http:// www.monografias.com/trabajos 11/pagnaweb/ pagnaweb.shtml

Ayala, J. y De Paúl, J. (2004). Trastorno de estrés postraumático en víctimas de situaciones traumáticas. Psicothema, 1, 45-49.

Barraza, B. (2000). Violencia en los medios de comunicación. Revista Salvadoreña Pixeles Cuscatlecos. 2005. Recuperado el 13 de julio de 2005 de hhtp://wwwpixelescuscatlecos.com/ psicologia

Berkowitz, I. (1996). Agresión: causas, consecuencias y control. Madrid: Desclee de Brouwer.

Berman S., Silverman W., y Kurtines W. (2000). Children 's and adolescents exposure to community violence. The australasian Journal of disaster and trauma studies. Recuperado el 14 de Julio de 2005 de http://www.massey.ac.nz/trauma/ issues/2001/berman.

Briere, J. (1996). Listado de síntomas traumáticos para niños. Professional Manual. Florida: Psychological Assessment Resources Inc.

Buchman, D. D., Funk, J. B., y Germann, J. N. (1997). Juegos de videos: investigación, puntajes $y$ recomendaciones. En: Cesarone, B. Recuperado el 12 de octubre de 2005 de http://www.ericdigests. org/2001-3/videos.htm
Buka, S., Birdthistle, I., Earls, F., y Stichick, T. (2001). Youth exposure to violence: Prevalence, risks, and consequences. En B. Stein, L. Jaycox, S. Kataoka, H.Rhodes, y K. Vestal. Prevalence of Child and Adolescent Exposure to Community Violence. Clinical Child and Family Psychology Review, 4, 76-82

Caballero, R., Mojarro, M., y Rodríguez-Sacristán, J. (1998). El estrés postraumático. En J. Rodríguez Sacristán. (ED.). En Psicopatología del niño y del adolescente. Sevilla: Universidad de Sevilla.

Cabezas A., Haquin F., y Larraguibel Q. 2004). Factores protectores y de riesgo en salud mental en niños y adolescentes de la ciudad de Calama. Revista chilena de pediatría. 75, 425-433. Recuperado el 4 de noviembre de 2005 de http:// www.scielo.cl/scielo.php?script

Campbell, C., y Schwarz, D. (1996). Prevalence and impact of exposure to interpersonal violence among suburban and urban middle school students. En B. Stein, L. Jaycox, S. Kataoka, H. Rhodes, y K. Vestal. Prevalence of Child and Adolescent Exposure to Community Violence. Clinical Child and Family Psychology Review, 4, 34-39

Cantor J. (1996). Children's fright reactions to television news. Journal of Communication. En González, J., Pérez, A.M., Pinzon, R y Sánchez, J. Influencia de la televisión violenta en niños de una escuela pública de Bogotá, Colombia. Revista salud pública, 1. Recuperado el 14 de julio de 2005 de http://www.scielosp.org/scielo.

Cantón, J., y Cortes, M. (1997). Malos tratos y abuso sexual infantil. Madrid: Siglo Veintiuno editores, S.A.

Kaiser Family Foundation. Center on Media and Child Health del Hospital Infantil de Boston (2003), publicado en 2005 por la, en la ZENIT (Agencia Internacional Católica de Noticias). Recuperado el 16 de Noviembre de 2005, de http://www.zenit.org/spanish/visualizza. phtml?sid=72425.

Centro de Investigaciones Médicas en Ansiedad. Recuperado el 22 de julio de 2005, de http:// www.centroima.com.ar/editoriales_02.asp

Presidencia de la Republica (2003). Niños podrán contar casos de maltrato a directora del ICBF. Recuperado el 13 de mayo de 2005 de www.presidencia.gov. co/ cne/2003/noviembre/20/14202003.htm - 8k -

Centro de Recursos de La Coalición de Indiana en Contra de la Violencia Doméstica (ICADV) (sf), Los efectos de la violencia en los niños. Recuperado el 18 de noviembre de 2005, de ttp://www. violenceresource.org/quietspan.pdf 
Cicchetti, D. et.al. (1993). Efectos de la violencia doméstica en problemas de conducta y depresión de los niños. Developmental Psychology, 29, 44 62.

Christoffel, K. (1990). Violent death and injury in US childrenand adolescents. En B. Stein, L. Jaycox, S. Kataoka, H. Rhodes, y K. Vestal. Prevalence of Child and Adolescent Exposureto Community Violence. Clinical Child and Family Psychology Review, 4, 45-48

Cogollo, Z., Campo, A., y Díaz, C. (2002). Niñas y niños desplazados que ameritan consulta psiquiátrica. Pediatría. Órgano oficial de la sociedad colombiana de pediatría, 2. Recuperado el 5 de agosto de 2005 de http://www.encolombia.com/medicina/ pediatria/pediatria37202-contenido.htm

Comisión de derechos humanos. (2001). Informe de la alta comisionada de las naciones unidas para los derechos humanos sobre la situación de los derechos humanos en Colombia. Recuperado el 8 de agosto de 2005, de http://www. womenscommission.org/pdf/co2_es.pdf.

Consultoría para los Derechos Humanos y el Desplazamiento. (2000). Desplazamiento forzado y derechos de la infancia. Boletín 25.

Costello, E., Erkanli, A., Fairbank, J. y Angold, A. (2002). The prevalence of potentially traumatic events in childhood and adolescence. Journal of Traumatic Stress, 15, 99-112.

Cova F., Maganto C., Valdivia M. (2005). Diferencias de género en psicopatología en la niñez: Hipótesis explicativas. Revista chilena de pediatría, 4. Recuperado el 11 de enero de 2006 de http://www.scielo. cl/scielo.php?pid=S037041062005000400014\&script=sci_arttext

Crouch, J. (2000). Violencia contra niños. Debats. 5, 7071.

Cuevas, M. (2002). Percepción de violencia entre escolares españoles y colombianos. Pontificia Universidad Javeriana-Cali.

Cuevas, M. (2004). Cuestionario de exposición a violencia. Investigación Psicología y Violencia de la Facultad de Humanidades y Ciencias Sociales de la Universidad Javeriana.

Cuevas M., y Gómez S. (2005) Proyecto de Investigación Intervención psicológica para el manejo de la ansiedad en niños expuestos a violencia. Colombia. Recuperado el 19 de noviembre de 2005, dehttp:// www.puj.edu.co/investigacion/6501_6505.htm.

Deas, M., y Gaitan, F. (1995). Dos ensayos especulativos sobre la violencia en Colombia. Tercer Mundo editores.
Del Barrio, M., y Moreno C. (1992). Acontecimientos vitales y depresión en adolescentes. Publicado en la revista IberPsicologia (1996) Recuperado el 12 de octubre de 2005 de http://fsmorente.filos. ucm.es/Publicaciones/lberpsicologia/iberpsi1/ moreno/moreno.htm

Díaz-Atienza F; Prados M; Ruiz-Veguilla M. (2004). Relación entre las conductas de intimidación, depresión e ideación suicida en adolescentes. Resultados preliminares. Granada. Recuperado el 10 de enero de 2006 de www.paidopsiquiatria. com/rev/numero4/art4.pdf

Díaz, F. (1998). Tratamiento psicológico de las víctimas del terrorismo. Conferencia en Foro Académico "Victimas y terrorismo", dictada en 1998 en la universidad Javeriana. Recuperado el 15 de octubre de 2005 de http://milton_leonardo_c. tripod.com/psicologiavirtual/publicaciones/ trtvictiterror.html

Dubner, A., y Motta, R. (1999). Sexually and physically abused foster care children and posttraumatic stress disorder. Journal of Consulting and Clinical Psychology, 67, 376-373.

Dubrow, Garbarino y Kosteleny. (1991). What children can tell us about living in danger. En: M. Jipguep, y Sanders- Phillips. The Context of violence for Children of Color: Violence in the Community and in the Media. Journal of Negro Education, 4. Recuperado el 21 de Julio de 2005, de la base de datos Proquest.

Finkelhor, D., Ormrod, R., Y Turner, H. (2006). The effect of lifetime victimization on the mental health of children and adolescents. Recuperado el 10 de nero de 2006 de la base de datos Proquest.

Foster, J., Kuperminc, G., y Price, A. (2004). Gender differences in posttraumatic stress and related symptoms among inner-cityminorityyouthexposed to community violence.1, 59-62 recuperado el 30 de noviembre de la base de datos Proquest.

Garbarino, J., y Kostelny, K. (1996). The effects of political violence on Palestinian children's beahaviour problems: A risk accumulation model. Child Development, 67, 33-45.

García, M. (2000). Televisión, Violencia e infancia: El impacto de los medios. Barcelona: Edit. Gedisa

Gil, D. (s.f.). Aumenta la ansiedad infantil. Recuperado el 17 de julio de http://

www.donbosco.es/universojoven/nivel3. asp? men=1\&submen=Escuela $\% 20$ de $\% 20$ Padres\&val=22\&page $=3$

Gil, D. (2005). Los jóvenes, más agresivos cuantos más videojuegos y diarios consumen. Diario 20 minutos. Recuperado el 13 de julio de 2005,dehttp:// 
www.20minutos.es/noticia/4286/o/jovenes/ television/videojuegos

Giraldo, J. (s.f.). Los pobres sometidos a los poderes de este mundo: desafíos al trabajo por la justicia en Colombia. Recuperado el 17 de julio de 2005, de http:// www.sedos.org/spanish/giraldo7.htm

Goldstein R., Wamper N, y Wise P. (1997). War experiences and distress symptoms of Bosnian children. En Allwood, Bell, Arshad. Children's trauma and adjustment reactions to violent and nonviolent war experiences. Journal of the American academy of child \& adolescent psychiatric, 41, 33-38. Recuperado el 22 de julio de http:// www.jaacap.com/ -

González R., Pérez I., Pinzón A., y Sánchez J. (2004). Influencia de la televisión violenta en niños de una escuela pública de Bogotá, Colombia. Salud pública, 1, 20-24. Recuperado el 14 de julio de 2005 de http://www.scielosp.org/scielo

Gordis, E. y Margolin, G., (2000). The effects of family and community violence on children. En: M. Jipguep y Sanders- Phillips. The Context of violence for Children of Color: Violence in the Community and in the Media. Journal of Negro Education, 4, 17-21. Recuperado el 21 de Julio de 2005, de la base de datos Proquest

Greenfield P., y Beagles-Roos J. (1998). Radio vs. Television: Their cognitive impact on children of different socioeconomic and ethnic groups. En I. Pérez, A.M. Pinzon, R. González, J. Sánchez. Influencia de la televisión violenta en niños de una escuela pública de Bogota, Colombia. Revista salud pública, 1, 30-34. Recuperado el 14 de julio de 2005 de http:

Groebel, J. (1998). ¡Cuidado! Los niños están viendo. Recuperado el 4 de mayo de 2005 de http://www. hinifiti.de/gaming/UNESCO.htm

Goguen, C. y Hamblen, J., (2003). Community Violence. Recuperado el 6 de agosto de 2005, de http:// www.ncptsd.va.gov/facts/specific/fs_comm _ violence.html

Hernández, E. (2005). Depresión infantil. Recuperado el 22 de julio de 2005, de http.// www. psicologoinfantil.com/articulodepresion.

Horowitz K., Mckay M., y Marshall, R. (s.f) Community violence and urban families: Experiences, effects and directions for intervention. Pubmed, national library of medicine 2005. Recuperado el 3 de agosto de 2005 de Ttp://www.ncbi.nlm.nih.gov

Horowitz, K., Jekel, J., y Weine, S.(1995). PTSD symptoms in urban adolescent girls: compounded community trauma. En: M. Jipguep y Sanders- Phillips. The Context of violence for Children of Color: Violence in the Community and in the Media. Journal of Negro Education, 4. Recuperado el 21 de Julio de 2005, de la base de datos Proquest.

Amnistía Internacional (2005). Informe Anual. Recuperado el 15 de diciembre de 2005 de http:// web.amnesty.org/report2005/index-esl -

Instituto Colombiano de Bienestar familiar. (2000). Recuperado el 3 de julio de 2005 de http://www. icbf.gov.co/espanol

Instituto Colombiano de Bienestar familiar. (2002). Recuperado el 15 de julio de 2005 de http://www. icbf.gov.co/espanol/Noticias3.asp?ldNot=16

Instituto Colombiano de Bienestar familiar. (2003). Recuperado el 22 de agosto de 2005, de http:// wwwicbf.gov.co/español/Noticias.

Instituto Nacional de Medicina Legal y Ciencias Forenses. (2002). Informe anual. Recuperado el 2 de julio de 2005 de http://www.medicinalegal. gov.co/paginanewz_crnv/4 \%20violencia \%20 intrafamiliar.pdf

Instituto Nacional de Medicina Legal y Ciencias Forenses. (2004). Informe anual. Recuperado el 13 de julio de http://www.medicinalegal.gov.co/ paginanewz_crnv/5 \%20delito \%20sexual.pdf

Iraheta, M. (2003) Psicotrauma en sobrevivientes de guerra en Guatemala. Recuperado el 13 de julio de 2005, de http:// www.scielo.sa.cr/scielo.

Jipguep, M. y Sanders- Phillips, K. (2003). The Context of violence for Children of Color: Violence in the Community and in the Media. Journal of Negro Education, 4 (72). Recuperado el 21 de Julio de 2005, de la base de datos Proquest.

Joiner Jr.,Laurent, J., y Schmidt, K., et al.(2002). Anxiety sensitivity as a specific and unique marker of anxious symptoms in youth psychiatric inpatients. Journal of abnormal child psychology, 2, 167176. Recuperado el 2 de agosto de 2005, de la base de datos Proquest.

Kaltiala-Heino, T. (sf). Recuperado el 15 de diciembre de 2005 de http://www.educar.org/articulos/ violencia.asp edicar.org y aprender.org

Kerlinger, F (2002). Investigación del comportamiento. (3a. Ed.) México D.F: McGraw-Hill Interamericana

Kovacks, M. (1981). Child Depression Inventory. Professional Manual.

Psychological Assessment Resources Inc.

Kuther, T. (1999). A developmental-contextual perspective on youth convictimization by community violence. Adolescence. RoslynHeights. 136, 699 - 712. Recuperado el 13 de diciembre de 2005 de la base de datos Proquest. 
León, O. y Montero, I (2003). Métodos de investigación en psicología y educación. Madrid: McGraw-Hill.

Linares, L.O. (2001). Community violence: The effects on children. En M. Jipguep y Sanders- Phillips. The Context of violence for Children of Color:Violence in the Community and in the Media. Journal of Negro Education, 4. Recuperado el 21 de Julio de 2005, de la base

López, L., Tirado V., Velarde G. (2003). Determinación del nivel de ansiedad escolar en un colegio privado. Recuperado el 10 de enero de 2006 de http:/ wwwacademico.uno.mx/biblioteca/metodos/

Mccloskey, L., y Stuewing, J. (2005). The Relation of Child Maltreatment to Shame and Guilt Among Adolescents: Psychological Routes to Depression and Delinquency. Recuperado el 22 de noviembre de 2005 de la base de datos Proquest.

Mayor F. (2001). I Congreso internacional sobre "Etica en los contenidos de los Medios de Comunicación e Internet. Granada Recuperado el 13 de julio de 2005 de http://www.ugr.es/ sevimeco/congreso. htm

Mazza, J., y Overstreet, S. (2000). Children and adolescents exposed to community violence: $A$ mental health perspective for school psychologists. En: M. Jipguep y Sanders- Phillips. The Context of violence for Children of Color: Violence in the Community and in the Media. Journal of Negro Education, 4. Recuperado el 21 de Julio de 2005, de la base de datos Proquest.

Matsui O., Meda M, y Sierra D. (2004). Relación entre la ansiedad y la depresión en niños escolares con superdotacion intelectual de 9 años de edad. Recuperado el 12 de octubre de 2005 de http://www.cucs.udg.mx/revista_salud/Revista \%20de \%20Educacion \%20y \%20Desarrollo/ RED_003/003_Red_Valadez.pdf

Merlo, T. (1994). ¿Porque consumimos violencia? Una visión interactiva de la T.V. y sus consumidores. Perspectivas sistémicas, 53, 37- 45

Muñoz, J. (1998). Medios de comunicación masiva y estrés postraumático. Psicología Iberoamericana, 1, 67-72.

O'Connor, M. (2001). An assessment tool for evaluating affect development and functioning among adjudicated adolescent boys in residental treatment. Smith College Studies in Social Work. Norhampton: 2 (72) 329 - 343. Recuperado el 3 de diciembre de 2005 de la base de datos proquest.

Organización Panamericana de la Salud. (1994). XXXVII reunión del Consejo directivo de la OPS.Pensar la violencia desde la salud pública. Informe especial. Recuperado el 25 de junio de 2005 de www.medicos-municipales.org.ar/informe0699. htm - 9k

Organización Panamericana de la Salud. (2002). Informe mundial sobre la violencia y la Salud. Resumen. Publicado en español por la Organización Panamericana de la Salud para la Organización Mundial de la Salud Washington.

Organización mundial de la salud. (2002) Informe mundial sobre la violencia y la salud. Ginebra. Recuperado el 18 de julio de 2005 de http://www. medicos-municipales.org.ar/informe0699.htm

Ortiz, D., y Chaskel, R. (2013). El impacto en niños y adolescentes de los eventos ligados al conflicto armado. Recuperado el 17de julio de 2015 de https://scp.com.co/precop/precop_files/ modulo_8_vin_3/Imp_ninos_adol_conf_ armado.pdf

Osofsky, J., Wewers, S., Hann, D., y Fick, A. (1993). Chronic community violence: What is happening to our children?. En B. Stein, L. Jaycox, S. Kataoka, H. Rhodes, H. y K.Vestal, K. Prevalence of Child and Adolescent Exposureto Community Violence. Clinical Child and Family Psychology Review, 4.

Osofsky, J. (1995). The effects off exposure to violence on young children. En: Hamblen, J. y Goguen, C. community Violence. Recuperado el 6 de agosto de 2005, de http://www.ncptsd.va.gov/facts/ specific/fs_comm_violence.html

Otaya, K. (s.f). Intervención psicoterapeutica con niños traumatizados por guerra. Recuperado el 12 de octubre de 2005 de http://www.ishhr.org/ conference/articles/otoya.pdf

Palacio J. (1997). Estrés postraumático y resistencia psicológica en jóvenes desplazados. Recuperado 14 de julio de 2005 de

http:// www.uninorte.edu.co/divisiones/ humanidades

Paramjit T., Shulamit M. (2004). Disaster, Terrorism, and Children. Psychiatric Annals, 34. Recuperado el 17 de abril de la base de datos Proquest.

Peña, G., y Varela, M. (2004). Impacto psicológico de la exposición indirecta a la violencia socio-política en los niños escolarizados de la ciudad de Cali. Tesis de grado. Carrera de psicología. Pontificia Universidad Javeriana-Cali.

Perry, B. (1999). Post-traumatic stress disorders in children and adolescents. Current Options in Pediatrics, 11.

Perry, B. (2002). The vortex of violence: How children adapt and survive in a violent world. En: M. Jipguep y Sanders- Phillips. The Context of violence for Children of Color: Violence in the Community 
and in the Media. Journal of Negro Education, 4. Recuperado el 21 de Julio de 2005, de la base de datos Proquest.

Piernavieja C. (2005). La agresividad se da más en quien más horas ve de televisión. Aula del mundo. Recuperado el 13 de julio de2005 de http:// aula. el-mundo.es./aula/noticia. Php

Plan Migración, Comunicación y Desarrollo (2004). Colombia, más allá de la migración refugio humanitario. La insignia. Recuperado el 17 de julio de www.lainsignia.org/sociedad.html - 42k

Policía metropolitana de Santiago de Cali (2005). Recuperado el 28 de julio de 2005 de http:// www.policia.gov.co/ -

Pynoos, R., Frederick, C., Nader K et al. (1999). Life treat and posstraumatic stress in school-age children. EnAllwood, Bell, Arshad. Children's trauma and adjustment reactions to violent and nonviolent war experiences. Journal of the American academy of child \& adolescent psychiatric, 41. Recuperado el 22 de Julio de http:// www.jaacap.com/ -

Pynoos, R. (2002). La Comisión Presidencial Nueva Libertad para la Salud Mental Reunión 11 de noviembre de 2002. Chicago. Recuperado el 15 de octubre de 2005 de http://www. mentalhealthcommission.gov/espanol/SP_ Meeting_Minutes_sept.htm

Reynolds, C., y Richmond, B. (1978). Escala revisada de Ansiedad Manifiesta para niños. Professional Manual. Psychological Assessment Resources Inc.

Revista la jornada (2005). Más de un cuarto de millón de desplazados en Colombia, 41 (1). Recuperado el 27 de octubre de 2005 de http://www.jotçrnada. unam.mx

Rodriguez, C. (2003). Parental Discipline and Abuse Potential Affects on Child Depression, Anxiety, and Attributions. Journal of Marriage and Family, 65, 809. Recuperado el 26 de julio de 2005 de la base de datos Proquest.

Rojas, E. (1998). La ansiedad: como diagnosticar y superar el estrés, las fobias y las obsesiones. Madrid: Ediciones Temas de Hoy.

Sáez, A. (2000). La Construcción Cultural de la Paz desde el Preescolar Violencia contra la Infancia en las Américas. Recuperado el 6 de agosto de 2005 de http://www.malostratos.com/contenido/ biblioteca/trabajos/violinfaname1.m

Servicio nacional de menores SENAME de Chile (2004). Estudio peritaje psicológico en abuso infantil. Recuperado el 15 de octubre de 2005 de http://www.sename.cl/interior/publicaciones/ estudio5.pdf
Smith, P., Perin, S., Yule, W., y Rabe-heskert, S. (2001). War exposure and meternal reactions in the psychological adjustment of children from Bosnia-herzegovina. En Allwood, Bell, Arshad. Children's trauma and adjustment reactions to violent and nonviolent war experiences. Journal of the American academy of child \& adolescent psychiatric, 41 (4). Recuperado el 22 de julio de http:// www.jaacap.com/ -

Sternberg, K., et. al. (1993). Efectos de la violencia doméstica en problemas de conducta y depresión de los niños. Developmental Psychology, 29 (1), 44-52.

Susser E., Jackson H., y Hoven C. (2001). Terrorism and mental health in school: the effects of september 2001 on New Cork City school children. En W. Fremont. Childhood reactions to terrorisminduced trauma: a review of the past 10 years. Journal of the American academy of child \& adolescent psychiatric,. 43, 381-392. Recuperado el 22 de julio de http:// www.jaacap.com/ -

Teubal, R. (2001). Violencia familiar, trabajo social e instituciones. Argentina: Paidós

UNICEF. (2001). UNICEF preocupado por las repercusiones de la violencia sobre los niños palestinos e israelíes. Comunicado de prensa.

UNICEF (2002). World Federtion for Mental Health. Los rostros del trauma y la violencia. Abuso domestico (o familiar): El trauma. Recuperado el 16 de noviembre de 2005 de

http://www.wfmh.org/wmhday/wmhdspanish2002/ sec1fvt_violence.htm.

UNICEF. (2005). Situación de la infancia. Recuperado el 25 de mayo de http://www.unicef.org.co/08abus.htm.2005.

Veenema, T. (2001). Children's exposure to community violence. En Jr. Schmidt, Laurent, et al.(2002). Anxiety sensitivity as a specific and unique marker of anxious symptoms in youth psychiatric inpatients. Journal of abnormal child psychology, 2, 167-176. Recuperado el 2 de agosto de 2005, de la base de datos Proquest.

Vermeiren, R. et al (2002). Exposure to violence and suicide risk in adolescents: A community study. Journal of Abnormal Child Psychology. New York. 5, 529 - 538. Recuperado el 27 de noviembre de 2005 de la base de Datos Proquest.

Wallach, L. (1996). The Clearing house on Early Education and parenting. Recuperado el 12 de noviembre de 2005 de http://ceep.crc.uiuc.edu/ eecearchive/digests/1996/viole96s.html 
